\title{
Description of Cartographic Heritage in Europeana Data Model
}

\author{
Albina Mościcka $^{\mathrm{a} *}$, Agnieszka Zwirowicz-Rutkowska ${ }^{\mathrm{a}}$ \\ ${ }^{a}$ Military University of Technology, Albina Mościcka, albina.moscicka@wat.edu.pl, Agnieszka Zwirowicz-Rutkowska, \\ agnieszka.zwirowicz@wat.edu.pl \\ * Corresponding author
}

Keywords: archival maps, cartographic heritage, metadata quality, Europeana Data Model

\begin{abstract}
:
Europeana is a portal that provides access to millions of objects from all kinds of cultural heritage communities. It enables users to find digital cultural works made available by cultural institutions throughout the European Union. Currently, Europeana provides access to a collection of more than 50 million books, periodicals, video clips, maps, photographs and digital documents from libraries, archives, museums and audio-visual archives in Europe. To facilitate Europeana's participation in the Semantic Web Europeana Data Model (EDM) has been developed. The idea of EDM is to constitute a framework for collecting, connecting and enriching metadata. It is a theoretical data model that allows data to be presented in different ways according to the practices of the various domains that contribute data to Europeana. EDM brings more meaningful links to Europe's cultural heritage data. Data from partners or external information resources with references to persons, places, subjects, etc., will connect to other initiatives and institutions. This will result in sharing enriched content, adding to it and thereby generating more content in ways that no single provider could achieve alone.
\end{abstract}

Presented research is the continuation of authors earlier study. In previous research, authors have proposed to integrate EDM structured information with geographic information system for movable heritage providing the semantic relations of movable heritage to the geographical space. This was a step into mixing GIS and Europeana world with the use of semantic contextualization for the object representations. Current research is the next step in this study and is going towards development of the new areas of EDM implementation - in cartographic heritage description.

The most famous part of cartographic heritage are archival maps, which were always present in the libraries and are also present in Europeana. Archival maps were always widely used in the research from many fields. The basis of these research is primarily collecting old maps, relevant to the subject matter of research, in terms of content, detail or topicality. The ability to reach such materials, which are scattered across numerous libraries or archives around the world, is crucial, and it determines whether it will be possible to undertake the planned research at all. Thus, one of the key tasks is to find materials that are comparable in terms of scale and that cover the same area and space in the historical period of interest.

In practice, maps have traditionally been treated as "unusual" books by librarians who catalogued them. This results in people describing metadata maps adjusting the description of the map to the book description, assigning information about the type, date of issue, access rights or language of the work. On the one hand, such information is typical of books, while on the other hand, it is most often located on each of the maps. People who describe digital library metadata resources do not have to be cartographers. The problem is even more serious as in archival cartographic documents, certain information may not be given directly, in a notation that is legible for a non-specialist. Second, even realizing the importance of the data needed to describe the map, without clear guidelines on where and how to save it in metadata, each operator will write it differently. This requires, however, the development of consistent rules for recording individual data and their consistent application.

Thus, a research problem has arisen as to whether the method of recording information about archival maps in digital libraries metadata allows users to find the desired cartographic material. Therefore, the aim of this research is to propose such a way of archival maps description in Europeana Data Model, that can be the basis for effective access to cartographic resources.

The access to the cartographic resources is mostly the result of metadata search and the aim of the effective access is to find the map that is necessary for our research. The quality of the metadata determines whether one is able to find the map that they are interested in or not. Quality is in this case understood as a set of features that determine how the product fits to satisfy certain needs. In the case of map description, it is a set of map properties or features that are stored in metadata. In other words, what kind of map-specific information should be saved in metadata (and in what way) to provide the proper characteristic of the spatially related object. 
Therefore, it was assumed that archival maps description in EDM should be compatible with the specified requirements for geographic information description, because archival maps are also the source of such information. The essence of the methodology used is to analyse set of classes and properties used in Europeana Data Model and indicate these which are compatible with the metadata elements provided by the ISO19115 standard, which are used to describe geographic information. The basic metadata elements required for the geographical description of an ISO 19115 data set have been adopted as the metadata scope pattern for archival maps. EDM classes and properties have been assigned to them. EDM class is a group of things that have common properties, where property is an element that expresses the relationship between two resources. Property can be seen as the attribute or characteristic of a resource.

As the result of the research, EDM properties, necessary to provide the proper characteristic of the archival maps are presented (edm:ProvidedCHO), together with EDM classes defined the way of saving each metadata element. According to the EDM rules, object described in EDM should be connected with the place in space by EDM class. The most important EDM classes are currently implemented core classes representing object and contextual classes that may be associated with it. Because EDM separates object from its digital representation, the core classes are to represent the core object. The contextual classes are provided to allow these entities to be modelled as separate entities from the object with their own properties if the data can support such treatment.

Contextual class important for geographic information purposes is edm:Place. It is defined as an area in space, in particular on the surface of the Earth, in the pure sense of physics: independent from temporal phenomena and matter. Places are identified by the content provider and named according to some vocabulary or local convention, and possibly normalized by Europeana at enrichment or at ingestion time. Places can be cities, regions, countries etc. edm:Place contains a list of properties describing the place, which can give us their basic characteristic. Information about places is recorded with the use of properties, such e.g. edm:currentLocation, edm:hasMet or edm:happenedAt.

The juxtaposition of classes and properties which fulfil ISO 19115 standard requirements have been presented with the use of Unified Modelling Language (UML). 